\title{
Belgeo
}

Revue belge de géographie

$1 \mid 2002$

Miscellaneous : Africa

\section{Pauvreté et marginalisation rurales en Afrique au sud du Sahara}

Poverty and rural marginalisation in Africa south of the Sahara

\section{Hugo Mwanza et Kabata Kabamba}

\section{(2) OpenEdition}

1 Journals

Édition électronique

URL : https://journals.openedition.org/belgeo/15423

DOI : 10.4000/belgeo.15423

ISSN : 2294-9135

Éditeur :

National Committee of Geography of Belgium, Société Royale Belge de Géographie

Édition imprimée

Date de publication : 30 mars 2002

Pagination : 3-16

ISSN : 1377-2368

\section{Référence électronique}

Hugo Mwanza et Kabata Kabamba, «Pauvreté et marginalisation rurales en Afrique au sud du Sahara », Belgeo [En ligne], 1 | 2002, mis en ligne le 31 mars 2002, consulté le 21 septembre 2021. URL : http://journals.openedition.org/belgeo/15423; DOI : https://doi.org/10.4000/belgeo.15423

Ce document a été généré automatiquement le 21 septembre 2021.

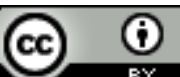

Belgeo est mis à disposition selon les termes de la licence Creative Commons Attribution 4.0 International. 


\section{Pauvreté et marginalisation rurales en Afrique au sud du Sahara}

Poverty and rural marginalisation in Africa south of the Sahara

Hugo Mwanza et Kabata Kabamba

\section{Introduction}

1 La progression de la pauvreté est un des phénomènes qui caractérisent l'évolution récente de l'Afrique, en particulier au sud du Sahara. Les effets de la crise pétrolière des années 1970, le désordre politique, la progression de la désertification, l'application des politiques d'ajustement structurel, etc. ont aggravé les conditions de vie des populations tant urbaines que rurales. D'où l'intérêt croissant qui a été accordé à la problématique de la pauvreté aussi bien par les acteurs de développement que par de nombreux chercheurs en sciences humaines et sociales. Pourtant, les campagnes africaines sont demeurées - hormis quelques enclaves exceptionnelles de relative prospérité - des parents pauvres des politiques d'investissement des différents Etats.

2 Signalons d'emblée que la définition de la pauvreté n'est pas fort aisée. Mais de nombreux analystes ont essayé d'aborder la question en l'inscrivant dans la problématique générale du sous-développement. Cependant, en ce qui concerne l'Afrique au sud du Sahara, quelle que soit l'échelle d'analyse et l'axe d'interprétation, la pauvreté n'échappe à aucune analyse.

3 La lecture rapide des indicateurs du PNUD ${ }^{1}$ met en évidence le fait que la pauvreté rurale ne peut être dissociée de la pauvreté générale de la région. L'article s'interroge surtout sur le devenir des campagnes au regard de certaines évolutions en cours. Cette analyse, faite au départ d'un certain nombre d'études disponibles sur l'Afrique centrale, semble malheureusement souligner un accroissement de la pauvreté et une plus grande marginalisation des campagnes. Un tel devenir constituerait à coup sûr, un véritable frein à la mise en place d'un réel processus de développement. 


\section{Etat des lieux de la pauvreté rurale en Afrique au Sud du Sahara}

\section{Lecture succincte de la pauvreté au travers des indicateurs de l'ONU}

Il n'est pas aisé d'établir un état des lieux de la pauvreté en Afrique, et en particulier en milieu rural, même si en Afrique subsaharienne la pauvreté se vit au quotidien, que ce soit dans les habitudes alimentaires, dans les comportements démographiques, dans les rapports sociaux entre hommes et femmes, etc. Ces faits sont difficilement quantifiables. Les seules données disponibles, en dehors des études locales, sont celles publiées dans les Rapports annuels des organismes internationaux (PNUD, FAO, etc.) ${ }^{2}$. Il s'agit en somme des estimations obtenues à partir des extrapolations et, de ce fait, elles ne peuvent refléter ni l'ampleur, ni la diversité géographique de la pauvreté dans des espaces pour l'essentiel en marge de l'économie-monde. Nous utiliserons ces données statistiques à titre indicatif pour surtout souligner la tendance à l'amplification du phénomène de pauvreté en Afrique subsaharienne.

5 En effet, au fil des Rapports du PNUD, on lit un accroissement de la pauvreté en général en Afrique subsaharienne. Ainsi, la population vivant au-dessous du seuil de pauvreté est passée de 179,6 millions en 1980 à 201,2 en 1990, à 218,6 en 1993 et à 291 millions en 1998. Pour 2000, on estime qu'une personne sur deux dans cette partie du continent vit au-dessous du seuil de pauvreté.

6 Pour rappel, au cours de la décennie 1980-1990, la population vivant en dessous du seuil de pauvreté en Afrique subsaharienne s'élevait déjà à $54 \%$ de la population totale. Les différentes données (Rapport sur l'Afrique publié par la CNUCED, 2001) ${ }^{3}$ montrent que l'Afrique subsaharienne est plus pauvre qu'il y a 20 ans du fait de la diminution de l'aide et de la dégradation des termes de l'échange, de l'accroissement de la dette et de l'inefficacité des politiques d'ajustement... En l'an 2000, en Afrique, le revenu par habitant était de $10 \%$ inférieur à celui de 1980 et ce sont les $20 \%$ les plus pauvres de la population qui ont été le plus durement frappés par deux décennies marquées par une croissance au-dessous de la norme, leurs revenus ayant chuté de $2 \%$ par an. Or, la plupart d'entre eux vivent dans les zones rurales.

7 Au cours de la même décennie (80-90), on estimait déjà que $65 \%$ de la population rurale contre seulement (c'est une façon de parler) $23 \%$ de la population urbaine, vivait audessous du seuil de pauvreté (CNUCED, 2001). Les différents indicateurs sociaux disponibles soulignent aussi cette misère rurale. En RDC par exemple, en 1995, la durée de vie moyenne en milieu urbain était de 56,6 ans alors qu'en milieu rural, elle s'élevait à 43,1 ans, soit un écart de 10 ans (RDC, DDK, 1998). Ce contraste ville-campagne apparaît assez nettement dans les statistiques de mortalité infantile (les décès d'enfants de moins d'un an) et de mortalité infanto-juvénile (les décès d'enfants de moins de 5 ans). Les taux moyens pour l'ensemble du territoire national sont élevés, soit respectivement 148 et 220 décès pour 1000. En considérant le milieu de résidence, ces taux se caractérisent par de fortes disparités, en ce sens que les taux de mortalité infantile sont de 101 p. mille en milieu urbain et de 161 p. mille en milieu rural. En ce qui concerne la mortalité infanto-juvénile, le taux est de $146 \mathrm{p}$. mille en milieu urbain pour 241 en milieu rural (id., 1998, p 60).

8 Les analyses récentes indiquent cependant une généralisation de la pauvreté en Afrique Subsaharienne quel que soit le milieu géographique. L'étude menée au Burundi en 1998 
(Rapport PNUD, 2000) indique que la part de la population vivant au-dessous du seuil de pauvreté est passée de $46 \%$ en 1990 à $48 \%$ en 1998 pour l'ensemble de la population burundaise. D'après le milieu géographique, on note un accroissement de la pauvreté en milieu rural où $56,4 \%$ de la population vivent au-dessous du seuil de pauvreté en 1998 contre $35 \%$ en 1990. En milieu urbain, ce phénomène, qui concernait $32 \%$ de la population en 1990, touche désormais près de $65 \%$ de la population.

9 Sans pour autant tomber dans l'afro-pessimisme, il y a lieu de s'interroger sur la pertinence des perspectives économiques prometteuses publiées annuellement par les organismes internationaux ${ }^{4}$ au sujet de l'Afrique. En s'appuyant sur les pronostics de la Banque mondiale pour évaluer la situation de la pauvreté en Afrique, Kappel (2000) estime que même en acceptant un taux de croissance très élevé du PIB de $7 \%$ par an et un meilleur accès de la population aux ressources (c'est-à-dire à leur redistribution), force est de constater que, même au bout de 15 ans, un grand nombre de personnes continueraient à vivre dans la pauvreté sur le continent. En admettant le taux un peu plus réaliste de $4 \%$, la pauvreté ne pourra être réduite que de façon marginale dans la plupart des pays. Avec un taux de croissance de $3 \%$, il ne sera pas possible de réduire le nombre des pauvres. Or, au cours des 20 dernières années, rares sont les pays qui ont pu enregistrer un taux de croissance de plus de $3 \%$. Si la tendance des deux dernières décennies se poursuit (taux moyen de croissance presque nul) la pauvreté va s'étendre encore davantage.

\section{Pauvreté rurale et crise socio-politico-économique en Afrique centrale}

La pauvreté rurale en Afrique subsaharienne est une des manifestations de la crise socio-politico-économique que traverse cette partie du continent depuis la fin des années 80. Des solutions préconisées ici et là pour faire face à la dérive de l'Afrique subsaharienne se sont soldées, à l'exemple des politiques d'ajustement structurel, par l'accroissement de la misère. Nous allons insister dans les limites de ce texte sur quelques éléments de cette crise multiforme et tenter de montrer dans quelle mesure, ces éléments contribuent à la marginalisation des milieux ruraux.

\section{Crise de l'état, dette et politiques d'ajustement structurel}

11 L'accroissement et la généralisation de la pauvreté en Afrique subsaharienne s'expliquent en grande partie par la crise de l'Etat. Celui-ci est remis en question depuis la fin des années 80 par les classes moyennes urbaines, en grande partie en raison de son incapacité à leur garantir une politique redistributive clientéliste.

Cette crise de l'Etat a eu des multiples effets pour la population, ne fût-ce que dans le fait que l'Etat consacre l'essentiel des moyens financiers au remboursement des intérêts de la dette. La solution préconisée par le FMI ${ }^{5}$, à savoir les plans d'ajustement structurel, visait à diminuer les dépenses sociales et à exporter, afin de rééquilibrer la balance commerciale et de récolter des devises fortes pour rembourser les intérêts de la dette. Le service annuel de la dette s'élevait en moyenne à 17 milliards de dollars US, soit l'équivalent de 3,8\% du PIB des pays, de $16 \%$ des exportations annuelles et de plus de $35 \%$ des dépenses d'éducation pour l'ensemble des pays de cette partie du continent. Malgré ces efforts, la dette extérieure de l'Afrique subsaharienne a presque 
quadruplé entre 1980 et 1999, passant de 60 à 231 milliards de dollars, alors que l'Afrique subsaharienne a remboursé au cours de la même période ce qu'elle devait au départ. Ces chiffres montrent à quel point la dette publique extérieure de l'Afrique subsaharienne constitue un obstacle à son développement. Elle empêche les progrès dans tous les secteurs, y compris l'éducation et la santé. On peut constater par ailleurs que le revenu moyen des citoyens d'Afrique subsaharienne a diminué de $25 \%$ au cours des vingt dernières années.

13 L'exemple du Gabon ${ }^{6}$ est assez caractéristique de cette problématique. Depuis 1986, ce pays traverse une situation de crise socio-économique liée à la chute du prix du pétrole et des produits de l'agriculture de rente (café, cacao,...). Le pays compte une énorme dette et consacre une part croissante du budget au remboursement des intérêts de la dette (43 \% en 1996). Entre 1985 et 1992, le nombre d'emplois dans les secteurs public et parastatal a diminué de $25 \%$, tandis que les postes à l'intérieur du secteur privé structuré baissaient de plus de $50 \%$ et de $18 \%$ dans le secteur pétrolier. On enregistrait en 1993 un taux de chômage de l'ordre de 22 \% à Libreville, de 38 \% à Port-Gentil et de $47 \%$ à Moanda parmi les chefs de ménage de moins de 27 ans. On assiste à une forte réduction des importations alimentaires (20\% des recettes pétrolières avant 1986). La dévaluation de $50 \%$ du franc CFA en 1994 a amplifié les effets de cette crise nationale et accentué la détérioration des conditions de vie des populations urbaines.

C'est surtout la crise pétrolière de 1986 qui a mis à nu les limites de cette économie rentière, car l'économie gabonaise est largement dépendante du pétrole. En 1970, le secteur pétrolier représentait $64 \%$ des exportations contre à peine $1,4 \%$ pour le secteur agricole. Cette dépendance s'est amplifiée au fil des années, de sorte qu'en 1985 ce secteur pétrolier représentait à lui seul $85 \%$ des exportations, $65 \%$ des recettes budgétaires et $45 \%$ du produit national brut. Malgré la crise du pétrole de 1986, la structure économique du pays a fort peu évolué. Le pétrole totalisait, en 1996, 79 \% des exportations, $58 \%$ des recettes de l'Etat et près de $45 \%$ des investissements bruts. Par contre la part de l'agriculture dans le PIB est en baisse constante : $24 \%$ en 1960 et à peine $3 \%$ en 1994 (Ministères de Finances, Programmation FMI, 1998). Les cours du cacao ont baissé de $50 \%$ entre 1974 et 1990, et la chute des cours mondiaux de café a atteint 40 \% entre 1989-1992, ce qui a entraîné la baisse de la production du cacao. Dans le Woleu-Ntem (plus de $70 \%$ de la production nationale) pour ne prendre que cet exemple, la production de café estimée à près de 6000 tonnes en 1960, à 5500 tonnes en 1974 ; s'élevait à 1600 tonnes en 1987 et à moins de 1000 tonnes en 1997. Malgré l'étatisation des circuits commerciaux de ces produits à la fin des années 70 (Pourtier, 1989), la situation n'a pu se redresser durablement.

Le pays se trouve par ailleurs confronté à une dette importante contractée en période de boom économique. Près de $45 \%$ du budget national a été consacré au service de la dette en 1997. Le poids des arriérés ( $+25,3 \%$ entre 96 et 97) et, dans une moindre mesure, des intérêts $(+4,7 \%)$ rendent cette charge difficile à assurer. Cela se traduit aussi par la chute des dépenses de fonctionnement et d'investissement, soit respectivement de $39,6 \%$ et $19,2 \%$ en 1996 à 37,8 \% et 18,4\% en 1997 (Ministères de Finances, Programmation FMI, 1998). 


\section{La pauvreté rurale dans la crise africaine} des écarts de niveau de vie entre citadins et ruraux avec une forte paupérisation urbaine et donc de nouveaux processus de socialisation. On peut se demander si la ville africaine apparaît toujours pour les ruraux comme un "mirage convoitable". Toutefois, cette crise a eu aussi des effets néfastes en milieu rural même si l'on en fait souvent peu de cas. Les campagnes déjà abandonnées à elles-mêmes s'enfoncent dans la misère, ne serait-ce qu'en raison du désengagement des pouvoirs publics des secteurs dits sociaux à l'exemple de l'enseignement. Certes, dans le cadre de la politique de la libéralisation de l'enseignement, on a vu éclore en milieu urbain des écoles privées, phénomène que, de toute évidence, on ne peut imaginer en milieu rural, peu propice à rentabiliser de tels investissements. L'Etat n'investit plus dans l'entretien de routes, accroissant l'enclavement des villages dont la plupart sont désormais sans intérêt pour les décideurs. Dès lors comment peut-on imaginer entreprendre en milieu rural, des programmes d'alphabétisation et de développement communautaire (eau potable, lutte contre les maladies parasitaires, etc.) ? d'une route ou de dragage d'un cours d'eau pour désenclaver un village si ce dernier ne présente pas un intérêt certain pour la ville, en termes d'approvisionnement alimentaire ou si on ne trouve pas dans cette campagne de productions minières ou agricoles à exploiter?

irmations se trouvent confortées récemment avec le développement des espaces périurbains qui, par leur proximité à la ville, fournissent aux centres urbains des produits alimentaires locaux à des prix concurrentiels, ne fût-ce que par les faibles coûts de transport. Ainsi, par exemple, autour de Yaoundé, à une distance de $100 \mathrm{~km}$, les coûts de transport équivalent à un prélèvement de $26 \%$ sur la production au lieu $\mathrm{de}^{7} \%$ à une distance de $10 \mathrm{~km}(\operatorname{CIRAD}(7), 1998)$. On verra par ailleurs que grâce aux opportunités qu'offrent les villes, les pratiques agricoles se sont nettement améliorées. Ainsi, les campagnes éloignées, enclavées n'ont plus rien à offrir : même le rôle de grenier agricole leur échappe.

\section{Le déclin de l'agriculture}

19 L'Afrique subsaharienne traverse une crise agricole persistante marquée par un déclin important des productions agricoles de rente (café, coton, exploitation forestière, etc.) et un recul sensible de la production vivrière qui ne parvient pas à répondre aux besoins d'une population de plus en plus urbanisée. Plusieurs raisons expliquent cette crise agricole, nous insisterons sur celles qui nous paraissent pertinentes et nous les illustrerons à partir des études de cas existantes.

\section{Prélèvements et affaiblissement des marges d'accumulation}

Une étude sur l'agriculture et le développement industriel au Burundi ${ }^{8}$ montre que la faiblesse des marges d'accumulation des exploitations paysannes est accentuée par l'intensité des prélèvements effectués sur le surplus agricole. Dans des pays non miniers surtout, ces prélèvements ont, avec le recours aux ressources externes, servi à financer le développement industriel. Au Burundi par exemple, si le pouvoir 
postcolonial a procédé à la suppression des cultures obligatoires, il les a remplacées par des journées de travail communautaire, organisées par le "Parti-Etat», durant lesquelles s'effectuent des travaux d'intérêt collectif. L'avènement de la II ${ }^{\text {ème }}$ République en 1976 a entraîné des réformes du système d'imposition avec la suppression de l'impôt de capitation et son remplacement par une épargne obligatoire sur trois ans (avec exception pour indigence) et l'abolition des formes traditionnelles d'exploitation. Enfin, le développement des échanges marchands a fait apparaître une nouvelle source de prélèvement à travers le système de prix, liée à la commercialisation par les paysans d'une partie de leur production. Ainsi, entre 1970-1987, la part des prélèvements sur les paysans a connu une hausse de près de $13 \%$. Ce taux peut paraitre, soulignent les auteurs, relativement faible par rapport à d'autres économies, mais ce qui est en jeu du point de vue de l'accumulation n'est pas tant la valeur absolue des prélèvements que l'importance par rapport au surplus. Si on examine la part des prélèvements dans le surplus agricole commercialisé, on constate que celle-ci accapare plus de $50 \%$ des revenus monétaires des paysans et dépasse, pour certaines années $60 \%$ de ces revenus. Au total, il est évident que cela réduit d'une manière substantielle les possibilités d'accumulation au niveau des exploitations. Par ailleurs $35 \%$ seulement des prélèvements étaient réinvestis dans l'agriculture. Or ces investissements au demeurant dérisoires sont effectués dans le sens du maintien du modèle de développement existant, et ils n'envisagent pas de réunir les conditions nécessaires à son dépassement (Hammouda \& Cochet, 1995, pp. 836-857). Au Rwanda, les dépenses publiques consacrées à l'agriculture n'atteignaient pas $5 \%$ du budget ordinaire de 1980-1997. Elles sont tombées de 4,3\% entre 1985-1987 à 1,2\% en moyenne entre 1995-1997. La part de l'agriculture dans le budget d'investissement a été en moyenne de $25,7 \%$ pour $1985-1987$ et à peine de $14,8 \%$ pour la période $1995-1997$ alors que le service de la dette atteignait $8,7 \%$ du budget ordinaire en 1995 et $11,16 \%$ en 1997, contre 1,7\% en 1995 et 1,2\% en 1997 consacré aux dépenses du secteur agricole (Banque Mondiale, 1998). On retrouve cette tendance dans le volume de la coopération affectée à l'agriculture par les membres de l'Union Européenne en Afrique subsaharienne. Le pourcentage de cette coopération qui représentait $21,7 \%$ du total, s'élevait à 14,9 \% en 1996 et à peine à 11,5\% en 1998.

\section{Le déclin des transports terrestres, l'exemple de l'état des transports routiers dans la province du Kasaï occidental en $\mathrm{RDC}^{9}$}

21 Au Kasaï, les routes sont généralement impraticables pendant une longue période de l'année correspondant à la saison des pluies (au moins 9 mois). Afin de mieux illustrer l'état des routes entre Kananga et sa région, nous nous limiterons à la description de la situation du Kasaï occidental. Les rapports publiés en 1992 par l'office des routes et le Service national des routes de desserte agricole (SNRDA) mettent en lumière les principaux aspects suivants :

- Les conditions d'accessibilité d'une grande partie de la province sont très difficiles : $22 \%$ des superficies seulement sont accessibles en permanence au cours de l'année, $28 \%$ sont accessibles temporairement pendant quelques mois et l'autre moitié (environ $75000 \mathrm{~km}^{2}$ ) est inaccessible ou très difficile d'accès en toute saison. Cette situation s'explique, entre autres, par l'état fortement dégradé des ponts et des bacs qui aggrave les conditions difficiles d'évacuation des produits vivriers vers les villes. En 1986, d'après l'office des routes, sur un total de 313 ponts, 44 (soit $14 \%$ ) seulement étaient en bon état, 3 (soit $1 \%$ ) 
dans un état assez bon et 266 (soit $85 \%$ ) en mauvais état. Quant aux bacs, sur un total de dixhuit, neuf seulement fonctionnaient.

- Ne sont en fait accessibles en permanence que les routes nationales, les routes régionales ainsi que les routes de desserte agricole situées dans l'arrière-pays proche de Kananga dans un rayon de 70 kilomètres. Les routes reliant Tshikapa aux principaux bourgs diamantifères de sa zone d'influence appartiennent aussi à cette catégorie.

22 Aux multiples problèmes de praticabilité du réseau routier s'ajoute celui de l'insuffisance et de la vétusté du charroi automobile. Sa réduction a été nettement remarquable au cours des quarante dernières années. Selon le rapport de la Division Régionale des Affaires Economiques à Kananga, 2973 véhicules appartenant à des sociétés privées étaient destinés à l'évacuation des produits vivriers en 1958. Au fil des années le charroi automobile destiné au transport des vivres a sensiblement diminué : en 1979-1980, on totalisait pour l'ensemble du Kasaï, 329 véhicules dont $24 \%$ seulement assuraient partiellement le transport de la production agricole vivrière ; $53 \%$ allaient au-delà de 100 kilomètres et, parmi eux, les $4 / 5$ effectuaient des liaisons interurbaines avec les villes diamantifères de Mbujimayi et Tshikapa.

Face à cette crise des transports terrestres, on a remarqué au Kasaï une extraordinaire capacité d'adaptation des acteurs économiques par l'adoption de nouvelles stratégies. Le rôle des transports aériens s'est sensiblement accru, particulièrement pour l'approvisionnement rapide et sûr du Kasaï en produits manufacturés. Les liaisons avec le reste du pays et même avec l'Afrique australe se font à partir des aéroports de Mbujimayi (devenu le deuxième aéroport du pays par l'importance du trafic), Kananga et Tshikapa. A l'aéroport de Mbujimayi, le tonnage du fret débarqué est ainsi passé, entre 1980 et 1989, de 1982 à 13806 tonnes; le nombre des passagers à l'arrivée qui était de 12092 en 1980 est passé à 36091 en 1989 et celui des passagers au départ est passé de 19534 en 1980 à 49620 en 1989. Cette tendance doit s'être sensiblement accrue au cours des dix dernières années si l'on considère les informations obtenues en avril 2000 auprès des services de l'aéroport de Mbujimayi : près de 100000 voyageurs y passent annuellement et environ 50000 tonnes de fret y sont débarqués. Pour Kananga, la tendance à la croissance du fret aérien a été forte également mais moins qu'à Mbujimayi : 5325 tonnes de fret débarqués en 1996 contre 2285 tonnes en 1980 et environ 40000 voyageurs. Ce recours à l'avion dans l'approvisionnement des villes du Kasaï, bien que présentant le double avantage de la rapidité et de la sûreté de livraison, a pour principal inconvénient l'enchérissement du prix de revient et, par conséquent, du prix de vente des produits de première nécessité.

$24 \mathrm{Au}$ total, toutes ces difficultés de transport ont sensiblement réduit l'aire régionale d'approvisionnement de Kananga et ont entraîné l'émergence de nombreuses stratégies d'adaptation à la crise. Parmi ces stratégies de ravitaillement, on citera :

-L'approvisionnement de la ville en produits frais et en charbon de bois par portage ou à vélo ;

- Le développement des activités agricoles dans l'arrière-pays immédiat de la ville, c'est-àdire l'espace rural situé au-delà de la zone périurbaine entre 15 et 40 kilomètres du centreville. La mise en valeur de cette zone périurbaine a eu un impact sur le foncier, les populations urbaines s'accaparant des terrains jadis villageois. Cela se fait par location ou tout simplement par achat.

- En milieu rural, les contraintes d'accessibilité obligent les paysans à une certaine tendance à l'autosubsistance là où ils ne sont pas sûrs de pouvoir écouler leurs productions. C'est le cas 
des paysans de tous les Territoires de la zone forestière au nord du Kasaï : Dekese, KatakoKombe, Kole, Lodja, Lomela, Lubefu et Lusambo. Ces contraintes ont aussi entraîné dans certains villages de la zone de savane du sud du Kasaï un changement de comportement des paysans-producteurs qui veulent à tout prix vendre leur production agricole. Lors d'un séjour dans le Territoire de Luiza au début des années 1990, nous avons constaté, par exemple, que certains paysans utilisaient des cossettes de manioc invendues comme combustible alors que d'autres venaient supplier les commerçants de passer par leur village pour acheter leur production, en offrant des commissions ${ }^{10}$. Cela mesure la fragile condition économique de paysans qui sont coupés des circuits monétaires et auxquels sont imposés des prix très bas.

\section{Le déclin démographique}

Le déclin démographique des campagnes est relatif. Les données statistiques disponibles montrent que la population rurale reste encore majoritaire dans plusieurs pays de l'Afrique au Sud du Sahara ${ }^{11}$. Elle représenterait, selon les estimations de la Banque Mondiale, $60 \%$ de la population totale.

Inscrite dans une perspective, la part de la population rurale est en constante régression. Elle est passée successivement de $85 \%$ en 1950, à $74 \%$ en 1975, 66 \% en 1995 et près de $60 \%$ en 2001. La réduction de la part de la population rurale est à mettre en rapport avec le contraste des taux de croissance annuels (tableau 1). Entre 1990 et 1995, excepté la $\mathrm{RDC}^{12}$, les écarts de croissance entre les villes et les campagnes dépassent en moyenne $3 \%$.

Tableau 1. Taux de croissance annuels en \% des populations urbaine et rurale entre 1990 et 1995.

\section{Population urbaine Population rurale}

$\begin{array}{lll}\text { Cameroun } & 4,9 & 1,2 \\ \text { RCA } & 3,1 & 1,7 \\ \text { Congo } & 4,8 & 0,7 \\ \text { RDC } & 4,4 & 3,7 \\ \text { Guinée Equatoriale } & 5,9 & 0,4 \\ \text { Gabon } & 5,2 & 0,7\end{array}$

Source : United Nations Population Information «POPIN »

Cette situation s'explique par l'exode rural. Au Gabon par exemple, la population urbaine représentait près de $73 \%$ de la population totale en $1993^{13}$ alors qu'elle était estimée à $20 \%$ à peine en 1960. Elle a crû en moyenne de 5,2\% par an entre 1990 et 1995. C'est l'un des taux de croissance annuelle les plus élevés en Afrique centrale (tableau 1). La croissance urbaine contraste fortement avec celle du milieu rural ( $0,7 \% /$ an). 

Une étude consacrée au Kwilu dans la Province de Bandundu (RDC) donne une moyenne de 84 hommes pour 100 femmes dans les villages et parfois seulement 60 (Nicolaï, 1994, p. 28). Ce phénomène de "féminisation" de la population rurale constituerait l'une des difficultés auxquelles se heurte la croissance de la production agricole, par manque de main-d'œuvre pour défricher la forêt puisque de nombreux hommes sont partis à la ville. La faiblesse de l'indice de masculinité en milieu rural est observable dans les données globales de la Province (tableau 2).

Tableau 2. Répartition de la population résidente de la Province de Bandundu par grands groupes d'âge et par sexe, selon le milieu (en milliers).

\begin{tabular}{|c|c|c|c|c|c|c|c|c|c|c|c|c|}
\hline \multirow{2}{*}{$\begin{array}{l}\text { Groupes } \\
\text { d'âge }\end{array}$} & \multicolumn{5}{|c|}{ URBAIN } & \multicolumn{7}{|c|}{ RURAL } \\
\hline & Masc. & $\%$ & Fém. & $\%$ & Total & $\%$ & Masc. & $\%$ & Fém. & $\%$ & Total & $\%$ \\
\hline $0-14$ & 2054 & 23,9 & 2066 & 24,0 & 4120 & 47,9 & 4995 & 22,6 & 4987 & 22,5 & 9928 & 45,1 \\
\hline $15-59$ & 2148 & 25,1 & 2123 & 24,7 & 4271 & 49,8 & 5150 & 23,1 & 5819 & 26,4 & 10980 & 49,5 \\
\hline $60 \mathrm{et}+$ & 117 & 1,2 & 96 & 1,1 & 213 & 2,3 & 593 & 2,8 & 583 & 2,6 & 1165 & 5,4 \\
\hline Total & 4319 & 50,2 & 4285 & 49,8 & 8604 & 100 & 10738 & 48,5 & 11389 & 51,5 & 22127 & 100 \\
\hline
\end{tabular}

INS, République du Zaïre, 1991

De façon globale, on note une forte proportion de jeunes et une faible importance de la population âgée quel que soit le milieu. Une analyse selon le sexe montre que la population masculine est relativement plus importante en milieu urbain $(50,2 \%)$ qu'en milieu rural $(48,5 \%)$. Autre constat, la population de 60 ans et + représente $5,4 \%$ en milieu rural contre seulement $2,3 \%$ en milieu urbain.

Une analyse détaillée selon le sexe indique que $26 \%$ des femmes sont dans la classe d'âge des 15-59 ans en milieu rural et seulement $23,1 \%$ des hommes. La situation est plus ou moins équilibrée (25,1\% des hommes et $24,7 \%$ des femmes) dans le milieu urbain. Pour les 60 ans et plus, les écarts selon le sexe sont très faibles, mais davantage selon le milieu de résidence. En effet, les 60 ans et plus représentent à peine 1,2\% des hommes et $1,1 \%$ des femmes en ville. Dans les campagnes, ces proportions sont deux 
fois supérieures $(2,8 \%$ des hommes et $2,6 \%$ des femmes). On assiste ainsi à un vieillissement relatif de la population rurale par le fait du départ des 15-59 ans et le retour des personnes plus âgées (pensionnées, malades...).

\section{Émergence et développement de nouveaux espaces ruraux}

Plusieurs nouveaux types d'espaces émergent, nous en étudierons deux exemples : les espaces périurbains et les zones d'exploitation minière artisanale.

\section{Les espaces périurbains}

La périurbanisation a pris ces dernières années une ampleur sans précédent dans les métropoles africaines. Ce phénomène est bien entendu moins marqué en ce qui concerne les villes moyennes, car il est fonction non seulement de la distance à l'agglomération mais aussi de la taille de celle-ci (Chaleard, 1996).

Contrairement à la vague des années 70 et 80 marquée davantage par le développement de l'habitat urbain, on assiste depuis la fin des années 80 à un redéploiement des activités socio-économiques dans ces espaces proches et/ou éloignés mais relativement bien reliés à la ville. Ce redéploiement s'accompagne de nouvelles techniques de production et d'exploitation des ressources, mais également de la marchandisation de la terre. Il en résulte de nombreux conflits que les autorités locales sont incapables de gérer.

Le phénomène de périurbanisation est en relation avec la forte crise alimentaire qui résulte de la crise de l'Etat. Les villes ont bénéficié dans la décennie 70 d'importants investissements, notamment en termes de subventions à l'importation de produits alimentaires. Cette politique de subvention qui se faisait au détriment des produits locaux a été remise en cause dans le cadre des plans d'ajustement structurel. Malheureusement, l'abandon des importations alimentaires subventionnées n'a pu contribuer à la promotion de l'agriculture locale en raison notamment de multiples problèmes d'accessibilité des campagnes.

C'est dans ce contexte qu'il faut situer le développement d'une agriculture périurbaine. Perçue au début comme un phénomène urbain de peu d'importance, l'agriculture périurbaine s'impose aujourd'hui comme un des greniers alimentaires des villes (CIRAD, 1998). Il est inquiétant de constater que cette agriculture est de plus en plus concurrencée sur le plan foncier par d'autres activités urbaines et par la multiplication de fermes appartenant à la bourgeoisie. Une grande partie de ces opérations dites agricoles relève d'une stratégie d'accaparement foncier qui se fait au détriment des associations paysannes.

Dans ces espaces périurbains, les opportunités économiques liées à la présence d'un marché de consommation, mais aussi à la possibilité d'investissements privés, participent à la mise en place des processus « d'intensification » agricole. Au Gabon, par exemple, on observe autour des villes, entre autres, la généralisation de l'usage de la tronçonneuse, le recours à la main-d'œuvre salariée dans les travaux agricoles, le raccourcissement de la durée de la jachère et l'extension du système de location des terres. 
39 Au niveau de la commercialisation, on note la tendance à la spécialisation et à la professionnalisation d'une catégorie de femmes commerçantes, les «Bayames " $^{15}$, spécialisées dans la commercialisation des produits agricoles en gros ou en demi-gros. Celles-ci peuvent compenser par l'importance des volumes concernés, la réduction de la marge bénéficiaire due aux difficultés de transport.

Cependant, l'« intensification» agricole ne concerne ni les milieux ruraux peu accessibles à la ville, ni toutes les couches de la population. Par exemple, la femme continue à assurer l'essentiel des activités de la production vivrière, elle s'occupe du semis et de l'entretien des champs, de la récolte et du portage des produits. Les activités de transformation (alcool de maïs ou de canne à sucre, etc.) lui incombent de même que la commercialisation des produits agricoles.

\section{Les nouveaux eldorados : espaces d'exploitation minière artisanale}

41 En RDC, les mesures de libéralisation des matières premières, définissant les règles d'exploitation des carrières minières, datent de $1981^{16}$. Ces mesures ont permis une multiplication des carrières. Dans la Province Orientale par exemple, Bilusa (1994) en totalisait 66 principales au 31 janvier 1994 ${ }^{17}$, Un élément significatif de leur importance est le volume total de carats extraits entre 1995 et 1998 par le secteur du diamant artisanal congolais soit 15 à 20 millions par an représentant une valeur commerciale estimée entre 600 et 650 millions de dollars. Ce qui place le secteur du diamant artisanal congolais parmi les gros producteurs mondiaux de diamant brut (en caratage) après l'Australie, selon les estimations fournies par H. Leclercq (2000, p. 59).

Une étude menée dans la zone de Tembo à la frontière entre la RDC et l'Angola souligne que 400 personnes y débarquent par mois et estime au total entre 25000 et 30000 les Congolais qui séjournent à Cafungo et aux alentours, en Angola (Sabakinu, 2000, p. 128).

Le centre de Kahemba situé dans le sud du Kwango-Kwilu (Nicolaï, 1996) est caractéristique d'un de ces espaces significatifs qui jalonnent les frontières de l'Afrique actuelle, là où les relations avec ce qui se trouve de l'autre côté de la frontière sont aussi fortes sinon plus fortes qu'avec le territoire national. Ce sont aussi des espaces de contrebande qui participent à l'économie informelle. Le transit et la contrebande du diamant angolais, en provenance de régions déchirées par la guerre civile et l'exploitation clandestine maintenant libéralisée, c'est-à-dire autorisée, de modestes gisements alluvionnaires locaux, ont assuré le développement de ce petit centre (quelques centaines d'habitants en 1960, 10000 en 1984) où règnent les trafiquants dans une sorte de Far West kwangolais.

Une enquête de 1995 auprès d'un échantillon de 100 personnes « bana Lunda » (c'est-àdire des hommes participant à la récolte et au trafic du diamant) (Sabakinu, 2000) indique que $86 \%$ d'entre elles étaient âgées de 15 à 30 ans, qu'il s'agissait essentiellement de célibataires (79\%), que $32 \%$ avaient au minimum un titre de fin d'études secondaires et que $50 \%$ provenaient des zones rurales.

On retrouve ces mêmes caractéristiques dans la description des «creuseurs " que fait Bilusa pour les «carrières » de la Province orientale. Selon lui « les creuseurs sont des jeunes gens robustes, adultes et valides, dont l'âge varie entre 25 ans et 40 ans...» (Omasombo, 2000, p. 83). 

que dans la Province Orientale plusieurs villages sont menacés d'extinction en territoires de Banalia, de Bafwasende et d'Ubundu. Tous les secteurs du territoire de Banalia ont des densités de population inférieures à celles de 1959; de même, à Bafwasende, le dépeuplement affecte cinq secteurs sur six et dans le territoire d'Ubundu, dix secteurs sur onze.

\section{Pauvreté et Marginalisation socio-politique des campagnes en Afrique Centrale}

On peut soutenir l'idée qu'une des causes de la marginalisation rurale en Afrique est la sous-représentation des populations rurales au sein des grands corps du pouvoir qu'ils soient politiques, économiques ou sociaux.

La représentativité des campagnes dans ces groupes est souvent confondue avec l'origine ethnique, tribale et régionale de leurs membres. Or, ces derniers résident principalement en ville, loin des contraintes des campagnes et perçoivent les problèmes de développement à travers la lorgnette du développement urbain. Pour la plupart de ces citadins, la ville est le moteur du développement. De ce fait, elle bénéficie prioritairement des investissements au détriment des campagnes et de l'agriculteur, bien que l'essentiel de la population réside en milieu rural et que les économies de la plupart des pays en développement soient principalement financées par les produits de l'agriculture. En outre, le plus souvent, le peu de moyens financiers destinés à l'agriculture sont en grande partie engloutis dans les tâches administratives. Ainsi par exemple, sur les huit millions de dollars nécessaires à la mise en route de travaux de terrain dans le cadre du Programme mondial de lutte contre la désertification, $80 \%$ ont été affectés à des dépenses administratives et à des activités de soutien et seulement 1,6 million de dollars ont servi à financer des projets concrets (Ayensu, p. 27). D'autres évaluations montrent qu'au cours des années 80, l'Afrique a consacré $37,8 \%$ du budget de l'agriculture à des dépenses d'administration contre 2,6 et $11,5 \%$ respectivement en Asie et en Amérique latine. Par ailleurs, la formation et la recherche agricoles se voient attribuer une portion bien congrue en Afrique avec 4,9\% et 5,3\% de ce même budget (Ayensu, id.).

est aussi pénalisé par les importations. En Afrique subsaharienne (1993), plus de $80 \%$ de la valeur totale des importations agricoles consistait en produits alimentaires, principalement blé, farine de blé, riz, sucre, produits laitiers, poisson et la viande. Ces importations rendues à terme indispensables par le changement des habitudes alimentaires que l'on note chez les urbains, notamment dans les classes moyennes du fait de l'acculturation et pour la grande masse des urbains du fait de leur faible coût. Au Nigeria par exemple, les importations alimentaires ont été multipliées par 5 entre 1975 et 1983 et ont représenté près de $23 \%$ de la consommation apparente. Cet accroissement des importations alimentaires va de pair avec une baisse des barrières douanières sur les céréales de 40 à $10 \%$ (Egg, 1985, p. 550) pour assurer l'approvisionnement des consommateurs urbains peu solvables. Ainsi, dans ce pays le blé et le riz américains se vendaient en moyenne à la moitié du prix payé aux producteurs locaux. 
'avènement du processus dit de "démocratisation» en Afrique risque encore de renforcer le pouvoir urbain. Une mesure qui pourrait aider les campagnes serait d'obliger les candidats aux postes de députés ruraux à y avoir leur résidence principale et à y résider en dehors des périodes de sessions parlementaires. Cette mesure symbolique montrerait, à notre avis, le poids que l'Etat accorde aux campagnes. Le processus de « démocratisation » en cours sur le continent pourrait aussi s'ancrer dans les milieux ruraux par l'instauration des mécanismes de décentralisation et de participation des ruraux dans la mise en place de projets dits de "développement " rural.

\section{BIBLIOGRAPHIE}

ALTERSIAL, CERED, MSA (1985), Nourrir les villes en Afrique sub-saharienne, L'Harmattan, coll. Villes et Entreprises, Paris, $421 \mathrm{p}$.

AYENSU E. S. (1987), « Par-delà la crise de l'agriculture africaine. Equilibrer conservation et développement », Ceres, 117, mai/juin 1987, pp. 13-32.

BANQUE MONDIALE (1998), Rapport sur la pauvreté au Rwanda : situation actualisée, Banque Mondiale - Ministère des Finances et de la Planification économique, Ministère du genre, de la famille et des affaires sociales. Ministère de l'agriculture et Réseau des femmes, 53 p. + Annexes.

BANQUE MONDIALE (1989), L'Afrique subsaharienne : de la crise à une croissance durable.

CAHIERS GÉOGRAPHIQUES DE ROUEN (1994), « Pauvreté et crises dans le monde tropical », 39, $233 \mathrm{p}$.

CENTRE DE COOPERATION INTERNATIONALE EN RECHERCHE AGRONOMIQUE POUR LE DEVELOPPEMENT (CIRAD) (1998), Agriculture périurbaine en Afrique subsaharienne, Ateliers du Colloque de Montpellier (20 au 24 avril 1998), Documents en ligne, CIRAD.

CHALEARD J.-L. (1996), Temps des villes, Temps des vivres. L'essor du vivrier marchand en Côte d'Ivoire, Paris, Karthala, 661 p.

CHOSSUDOVSKY M. (2000), La mondialisation de la pauvreté. La conséquence des réformes du FMI et de la Banque Mondiale, Montréal, Les éditions Ecosociété, 248 p.

COMITE POUR L'ANNULATION DE LA DETTE DU TIERS-MONDE (CADTM) (2000), Le Bateau ivre de la mondialisation, Bruxelles, Syllepse.

COQUERY-VIDROVITCH C. (s/d. de) (1991), Sociétés paysannes du Tiers-monde, Paris, L'Harmattan, $282 \mathrm{p}$.

DE HERT T., MARYSSE S. (1996), « L'économie informelle au Zaïre. (Sur)vie et pauvreté dans la période de transition », Cahiers africains, 21-22, Institut Africain-CEDAF, Paris, L'Harmattan,194 p. INSTITUT NATIONAL DE LA STATISTIQUE (INS), Résultats du recensement scientifique, République du Zaïre, 1991.

GOUROU P. (1991), L'Afrique tropicale. Nain ou géant agricole ?, Paris, Flammarion, 225 p. 
GUBRY et al., (1996), Le retour au village, une solution à la crise économique au Cameroun, L'HarmattanIFORD.

HAMMOUDA H. B., COCHET H. (1995), « Agriculture et développement industriel au Burundi », Revue Tiers-monde, XXXVI, 144, Paris, IEDES, pp. 836-857.

KABAMBA KABATA (2000), Relations à la ville et territorialité dans la campagne environnante de Kananga, Thèse de doctorat, Université de Liège, $179 \mathrm{p}$.

KALONJI NTALAJA (1999), La pauvreté en République Démocratique du Congo. Niveaux, tendances et caractéristiques, Rapport du Ministère du Plan et du Commerce, Kinshasa, 43 p.

LAURENT P.-J. (1999), « Déstabilisation des paysanneries du Nord Kivu : migrations, démocratisation et tenures ", Cahiers Africains, 39-40, Institut Africain-CEDAF, Paris, L'Harmattan, pp. 63-83.

KANKODE M., TOLLENS E. (2001), (éd.), Sécurité Alimentaire au Congo-Kinshasa, Production, Consommation et Survie, K.U. Leuven et l'Harmattan, Paris, 2001.

KAPPEL R. (2000), « Afrique - Illusions sur le développement », $D+C, 3 / 2001$.

MABOLOKO NGULAMBANGU C.E. et NICOLAÏ H (2001), « Frontière, diamant et pauvreté. Le cas de la frontière Congo-Angola au Kwango », Revue Belge de Géographie, 1999/4, pp. 255-275.

MASHINI DHI MBITA M. (1994), Développement et stratégies spatiales dans le Kwango-Kwilu (sud-ouest du Zaïre), thèse de doctorat, inédite, Université Libre de Bruxelles, 3 vol. 636 p.

MBEMBE A. (2000), De la Postcolonie. Essai sur l'imagination politique dans l'Afrique contemporaine, Les Afriques, Paris, Karthala, 293 p.

MONNIER L., JEWSIEWICKI B. et VILLERS de G. (s/d. de) (2000), « Chasse au diamant au Congo/ Zaïre », Cahiers africains, 45-46, Institut Africain-CEDAF, Paris, L’Harmattan, 240 p.

MWANZA WA M. (1997), « Monocultures et dépendances. Le cas de la Côte d'Ivoire », Revue Défissud, Edition spéciale, n²9, pp. 33-34.

MWANZA M. et KABAMBA K. (1999), « Déclin des transports terrestres et difficultés d'approvisionnement de Kananga $(\mathrm{RDC})$ », in La route en Forêt tropicale : porte ouverte sur l'avenir ?, APFT, Working Paper, octobre 1999, pp. 53-56.

MWANZA M., TREFON Th., WOLFF E., (2001), Mutations socio-économiques et évolution des pratiques agricoles dans les espaces autour des villes moyennes en Afrique subsaharienne. Le cas gabonais, Université de Liège, Laboratoire d'Anthropologie de la Communication (sous presse).

NICOLAÏ H. (1992), «Les mutations récentes des espaces africains », Bulletin des séances de l'Académie royale des Sciences d'Outre-mer, 38, 4, pp. 563-573.

NICOLAÏ H. (1996), « Les transformations d'un espace africain : Le Kwilu. Réflexions sur le phénomène régional en Afrique centrale ", Bulletin de la Soc. Belge d'Etudes Géographiques - SOBEG, 1996-1, pp. 15-33.

POURTIER R. (1989), Le Gabon. Tome 2 : Etat et développement, Paris, L'Harmattan, 344 p.

Rapports du Programme des Nations Unies pour le Développement (PNUD) sur le Développement Humain.

RDC, Université de Kinshasa, Faculté des Sciences Economiques, Département de Démographie, La question démographique en RDC, UNIKIN, FUAP, Kinshasa, mai 1998. 
SINGARAVELOU (s/d. de) (1989), Pauvreté et Développement dans les pays tropicaux, Hommage à Guy Lasserre, CEGET-CNRS-GRET, Bordeaux, 585 p.

VENNETIER P. (s/d. de) (1989), « La périurbanisation dans les pays tropicaux », CEGET-Espaces Tropicaux, Espaces Tropicaux, 1, 383 p.

WOLFF E., TREFON Th., MWANZA WA M., SINTZOFF M. (1998), Géographie des Relations Ville-Forêt, Analyse à l'échelle locale, Rapport du Projet Programme Régional de l'Afrique Centrale pour l'Environnement (CARPE).

WOLFF E., TREFON Th., SINTZOFF M. et MWANZA WA M. (2002), « Des villes qui grignotent la forêt au Gabon », Revue Belge de Géographie, 1999/4, pp. 277-293.

\section{NOTES}

1. Programme des Nations Unies pour le Développement (PNUD).

2. Organisation des Nations Unies pour l'Alimentation et l'Agriculture (FAO).

3. Conférence des Nations Unies sur le Commerce et le Développement (CNUCED).

4. A titre d'exemple, Banque Mondiale (1989), L'Afrique subsaharienne: de la crise à une croissance durable.

5. Fonds Monétaire International (FMI).

6. MWANZA, WOLFF E., TREFON Th., SINTZOFF M. (1998), Géographie des Relations Ville-Forêt, Analyse à l'échelle locale, Projet Programme Régional de l'Afrique Centrale pour l'Environnement (CARPE).

7. Centre de Coopération Internationale en Recherche Agronomique pour le Développement (CIRAD).

8. HAMMOUDA H. B. et COCHET H., 1995.

9. Mwanza M. et Kabamba K., 1999.

10. Deux ou trois sacs de manioc remis par le chef du village.

11. Certains pays comme le Gabon et le Congo-Brazzaville sont devenus majoritairement urbains. Il faut toutefois, rester attentif au fait que la définition d'une ville varie d'un pays à l'autre.

12. Plusieurs facteurs seraient à la base de ce particularisme congolais : les longues distances entre villes et villages et les difficultés de transport, crise socio-économique généralisée, migrations plus marquée de petites villes vers les grands centres...

13. INS (1993), Recensement général de la population et de l'habitat. Si l'on considère le seuil officiel de 3000 habitants, $73 \%$ de la population gabonaise résidait en ville (définie comme une agglomération de plus de 3000 habitants) selon le recensement de la population de 1993. Si l'on considère les villes de plus de 10000 habitants en 1990, ce taux est de $45 \%$ au moins.

14. Recensements 1970, 1993.

15. Ces femmes commerçantes résident en ville.

16. Lire «Chasse au diamant au Congo/Zaïre» CEDAF, $n^{\circ} 45-46,2000$.

17. Aucune carrière du territoire d'Isangi n'était prise en compte. 


\section{RÉSUMÉS}

Le défi du développement en Afrique est indissociable du devenir des espaces ruraux. Ces derniers connaissent, à l'image de l'ensemble du continent, une crise sans précédent. On y observe une plus forte augmentation du nombre de personnes vivant dans la pauvreté que dans les villes, et une nette tendance au retour à l'autosubsistance.

La fin de l'Etat-providence marquant le désengagement des pouvoirs publics de secteurs sociaux, la marginalisation socio-économique des campagnes dans un contexte où le processus de développement n'est perçu que sous la lorgnette du développement urbain, l'émergence de nouveaux espaces concurrents à l'exemple des espaces périurbains et des espaces d'exploitation minière artisanale... sont autant de facteurs qui peuvent expliquer le déclin villageois. Celui-ci est lisible dans le déclin agricole, dans le déclin démographique, dans l'enclavement des espaces ruraux...

Un tel devenir constituerait à coup sûr un véritable frein à la mise en place d'un réel processus de développement.

The challenge of development in Africa goes hand in hand with the becoming of rural areas. The latter, just like the whole African continent, are faced with an unprecedented crisis and the increase in the number of poor people is higher there than in the cities. Rural areas are also experiencing a definite trend toward a return to self-sufficiency.

The end of the welfare state that marked the State disengagement from the social sectors, the socio-economic marginalisation of the rural areas in a framework within which development is considered in terms of urban development, the emergence of new rival spaces such as periurban areas and artisanal mining areas... are just so many factors that can account for the rural decline as expressed in the agricultural and demographic decline, in the remoteness of the rural areas... If it goes on this way, the setting up of a real development process will undoubtedly be curbed.

\section{INDEX}

Mots-clés : Afrique subsaharienne, pauvreté rurale, crise socio-économique, nouveaux espaces ruraux, marginalisation socio-politique

Keywords : Subsaharan Africa, rural poverty, socio-economic crisis, new rural areas, sociopolitical marginalisation

\section{AUTEURS}

\section{HUGO MWANZA}

Université Libre de Bruxelles, IGEAT, CP 246 bd du Triomphe, 1050 Bruxelles

\section{KABATA KABAMBA}

Institut Supérieur Pédagogique de Kananga (RDC) 